\title{
Enhanced Flow in Small-World Networks
}

\author{
Cláudio L. N. Oliveira, ${ }^{1,2, *}$ Pablo A. Morais, ${ }^{1, \dagger}$ André A. Moreira, ${ }^{1, \$}$ and José S. Andrade, Jr. ${ }^{1,}$ \\ ${ }^{1}$ Departamento de Física, Universidade Federal do Ceará, 60451-970 Fortaleza, Ceará, Brazil \\ ${ }^{2}$ Department of Biomedical Engineering, Boston University, Boston, Massachusetts 02215, USA
}

(Received 30 August 2013; published 9 April 2014)

\begin{abstract}
The proper addition of shortcuts to a regular substrate can lead to the formation of a complex network with a highly efficient structure for navigation [J. M. Kleinberg, Nature 406, 845 (2000)]. Here we show that enhanced flow properties can also be observed in these small-world topologies. Precisely, our model is a network built from an underlying regular lattice over which long-range connections are randomly added according to the probability, $\boldsymbol{P}_{i j} \sim \boldsymbol{r}_{i j}^{-\boldsymbol{\alpha}}$, where $\boldsymbol{r}_{\boldsymbol{i}}$ is the Manhattan distance between nodes $\boldsymbol{i}$ and $\boldsymbol{j}$, and the exponent $\boldsymbol{\alpha}$ is a controlling parameter. The mean two-point global conductance of the system is computed by considering that each link has a local conductance given by $\boldsymbol{g}_{i j} \propto \boldsymbol{r}_{\boldsymbol{i j}}^{-\boldsymbol{C}}$, where $\boldsymbol{C}$ determines the extent of the geographical limitations (costs) on the long-range connections. Our results show that the best flow conditions are obtained for $\boldsymbol{C}=0$ with $\boldsymbol{\alpha}=0$, while for $\boldsymbol{C} \gg 1$ the overall conductance always increases with $\boldsymbol{\alpha}$. For $\boldsymbol{C} \approx 1, \boldsymbol{\alpha}=\boldsymbol{d}$ becomes the optimal exponent, where $\boldsymbol{d}$ is the topological dimension of the substrate. Interestingly, this exponent is identical to the one obtained for optimal navigation in small-world networks using decentralized algorithms.
\end{abstract}

DOI: 10.1103/PhysRevLett.112.148701

PACS numbers: 89.75.Hc, 02.50.-r, 05.60.Cd

The Laplacian matrix operator is a general description for systems presenting two essential properties: (i) they obey local conservation of some load, and (ii) their currents of load are linearly dependent on some field [1]. Since such conditions are very often observed, this operator can be applied to several different problems in Physics, including diffusion [2,3], wave propagation [4], solving the Schrödinger equation in arbitrary graphs [5], dielectric breakdown [6], brittle fracture [7,8], Darcy's flow [9] and classical electrical transport [10,11], among others. In these problems, one is frequently interested in the stationary state, where currents in each edge of a given network can be determined using local conservation laws. In the field of complex networks, in particular, the Laplacian operator has been employed as a conceptual approach for determining the nature of the community structure in the networks [12], in the context of network synchronization [13], as well as to study network flow [14-19].

Given a regular network as an underlying substrate, it has been shown that the addition of a small set of random longrange links can greatly reduce the shortest paths among its sites. In particular, if the average shortest path $\ell_{s}$ grows slowly with the network size $N$ but the local clustering coefficient $C_{s}$ grows fast, typically when $\ell_{s} \sim \log (N)$ and $C_{s} \gg N^{-1}$, the network is called a small world [20-22]. If one considers the effect of constraining the allocation of the long-range connections with a probability decaying with the distance, $P_{i j} \sim r_{i j}^{-\alpha}$, results in an effective dimensionality for the shortest paths that depends on the value of $\alpha$ [23]. For the case in which the regular underlying lattice is one-dimensional, the small-world behavior has only been detected for $\alpha<2$, with $\ell_{s}$ reaching a minimum at $\alpha_{\text {opt }}=0$
[23]. The two-dimensional case was also investigated [24], yielding similar results.

The situation is much more complex if one does not have the global information of all the short-cuts present in the network. As a consequence, the traveler does not have a priori knowledge of the shortest path. Optimal navigation with local knowledge and the presence of long-range links was studied by Kleinberg [25]. Surprisingly, the small-world features of the network can only be efficiently accessed if the exponent is precisely set at $\alpha=d$, where $d$ is the topological dimension of the substrate. It is then claimed that this condition is optimal due to the presence of strong correlations between the structure of the long-range connections and the underlying lattice, leading to the formation of "information gradients" that allow the traveler to find the target. Later, it was shown that, by imposing a cost constraint to the long-range connections, results in $\alpha_{\text {opt }}=d+1$, for both local and global knowledge conditions [26].

A question that naturally arises from these navigation studies is how efficient small-world networks are for transport phenomena that typically obey local conservation laws. Here we show that enhanced Laplacian flow properties can also be observed for networks built by adding long-range connections to an underlying regular lattice, in the same fashion as previously proposed for navigation through small-world geometries [24-28]. Our model consists of $N$ nodes arranged on a circle, and connected to their two nearest neighbors. Long-range connections [29] are added to the regular substrate by ensuring that each node $i$ receives a new link to a node $j$ randomly chosen among those $N-3$ remaining nodes according to the probability, $P_{i j} \sim r_{i j}^{-\alpha}$, where $\alpha$ is an arbitrary exponent, and $r_{i j}$ is the Manhattan 
distance, namely, the minimum number of links separating nodes $i$ and $j$. The larger the parameter $\alpha$, the shorter are the long-range links. In fact, given this power-law distribution, for $\alpha<d$, the average link length grows with the system size, $\langle r\rangle \sim N$, while for $d<\alpha<d+1,\langle r\rangle \sim N^{d+1-\alpha}$, and for $\alpha>d+1,\langle r\rangle \sim N^{0}$. In the limiting cases, one may expect a logarithmic dependence, $\langle r\rangle \sim N / \log (N)$ for $\alpha=d$, and $\langle r\rangle \sim \log (N)$ for $\alpha=d+1$.

Once the network is built, we associate each link to an Ohmic resistor and a unitary global current is induced between a pair of sites $A$ and $B$ in the system (see Fig. 1). In order to compute the local potential $V_{i}$, we solve Kirchhoff's law at each site $i$ [30],

$$
\sum_{j} g_{i j}\left(V_{i}-V_{j}\right)=0, \quad i=1, \ldots, N,
$$

with the summation running over all sites connected to $i$, and $g_{i j}$ being the link conductance between $i$ and $j$. The inlet and outlet currents are also considered in the calculation of the local potentials at sites $A$ and $B$, respectively. The global conductance for a given realization, which depends on the chosen pair of sites $A$ and $B$, is computed as $G \equiv 1 / \Delta V$, where $\Delta V=V_{A}-V_{B}$, so that a mean global conductance $\langle G\rangle$, between any two sites of the network, is then obtained by averaging over different pairs of sites [31] and different realizations of the network.

Here we assume that the conductance of each link depends on the distance between its ends in the form,

$$
g_{i j}=r_{i j}^{-C},
$$

where the exponent $C$ gauges the way long-range connections impact the flow, as $C$ increases the contribution of

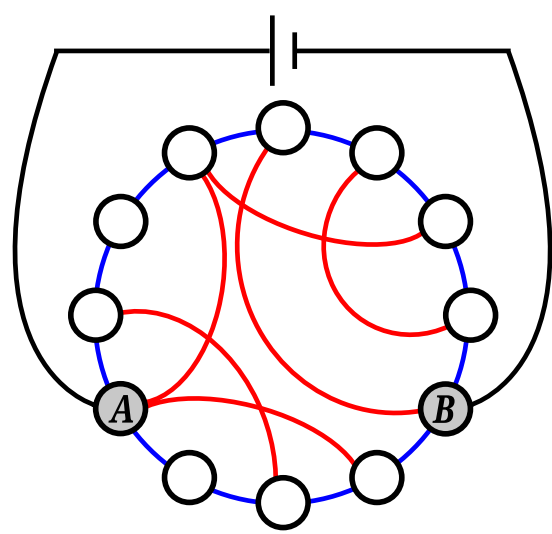

FIG. 1 (color online). Network built on a one-dimensional underlying lattice of nearest neighbors (short-range) connections (in blue) and long-range connections (in red). Each connection has a local conductance given by $g_{i j}$. A unity global current is applied through the system between nodes $A$ and $B$ so that a global conductance, $G_{A B}$, can be computed. We obtain the average conductance, $\langle G\rangle$, by calculating $G_{A B}$ over different pairs of sites $A B$ and several network realizations. longer connections to flow is attenuated. We focus primarily on two particular cases, namely, $C=0$ and 1 , but other cases with different values of $C$ are also investigated here. In the case of $C=1$, known as Pouillet's law [32], the conductance of a long-range connection with Manhattan distance $r$ is equivalent to an effective conductance of $r$ short-range links in series, hence one should expect longrange links contributing to transport as much as short-range links. Accordingly, for $C<1(C>1)$, preferential flow should occur through long-range (short-range) links.

As shown in Fig. 2 (inset), the network conductance $\langle G\rangle$ decays monotonically with $\alpha$ for $C=0$, regardless of the system size $N$, since the shortcut links in this case participate very actively in the flow. The maximum conductance is therefore obtained at $\alpha=0$. The main panel of Fig. 2 shows, however, that the average conductance calculated for $C=1$ behaves nonmonotonically with the exponent $\alpha$, with a maximum value observed at $\alpha=1$.

In Figs. 3(a) and 3(b) we show the dependence of the average network conductance on the network size $N$ for $C=1$ and 0, respectively. The results in Fig. 3(a) indicate that, except for $\alpha=1$, a typical power-law behavior is observed, $\langle G\rangle \sim N^{-\beta}$, with an exponent $\beta$ that depends strongly on the parameter $\alpha$ controlling the length of longrange connections. In this case, the local conductance is inversely proportional to the distance. As presented in the inset of Fig. 3(a), the exponent $\beta$ starts from 0.46, at $\alpha=0$, and falls to a minimum as $\alpha$ approaches unity. For $\alpha>1$, the exponent $\beta$ again grows continuously. In the limit of large values of $\alpha$, the added links always connect close sites, $\langle r\rangle \sim N^{0}$. As a consequence, the system should behave as a regular lattice, $\langle G\rangle \sim N / \log (N)$, regardless of the value of $C$. In the case of $C=0$, all links have identical resistances and a power-law behavior is always observed, as shown in Fig. 3(b). The inset of Fig. 3(b)

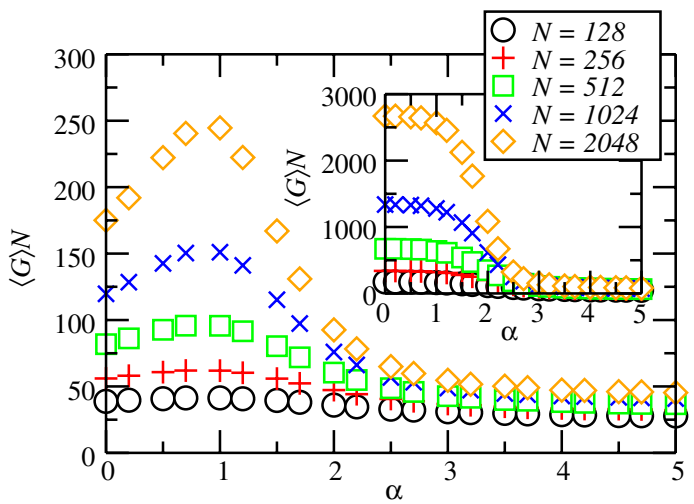

FIG. 2 (color online). Mean effective conductance versus the exponent $\alpha$ for $C=1$ (main graph) and $C=0$ (inset), in onedimensional substrates, and for different system sizes. The maximum conductance is obtained at $\alpha=1$, in the first case (local conductances inversely proportional to the length), and at $\alpha=0$, in the second case (conductances are independent of link length). The error bars are smaller than the symbols. 

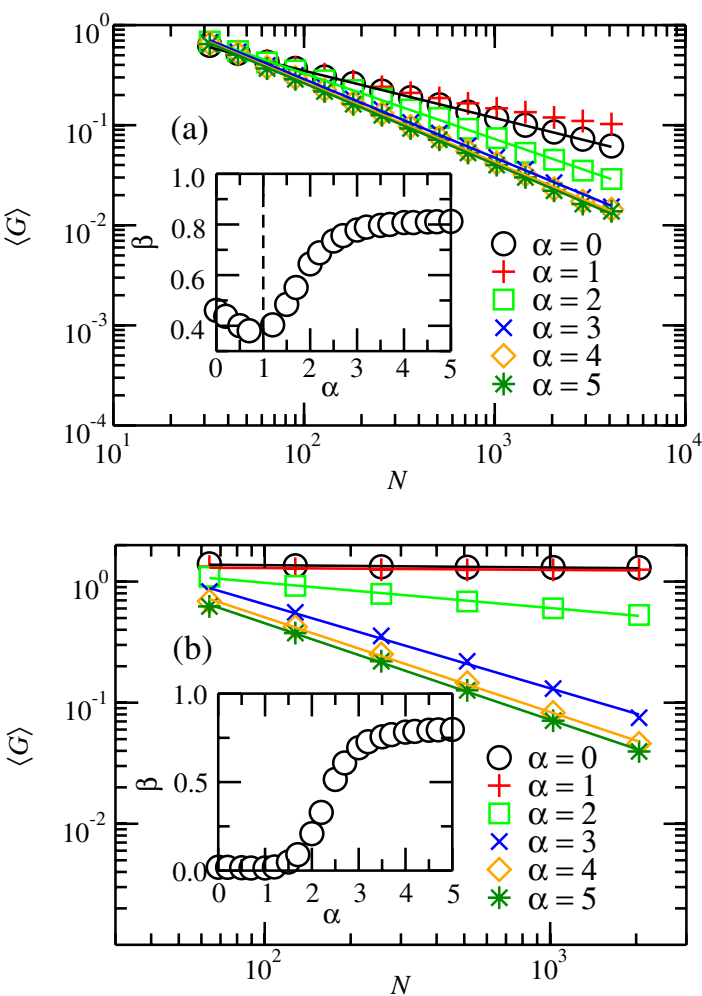

FIG. 3 (color online). Dependence on size of the mean effective conductance for different values of $\alpha$. In (a) we show the case in which local conductances are inversely proportional to the length $(C=1)$. The average conductances generally follow a power-law behavior, $\langle G\rangle \sim N^{-\beta}$, except for the optimal condition $\alpha=1$, where $\langle G\rangle$ behaves approximately as a power law of the logarithm of $N$, as depicted in Fig. 4. The exponents resulting from the least-squares fitting to the data of the scaling functions are shown in the inset, except for the case $\alpha=1$ (dashed line). A nonmonotonic behavior can be clearly observed. The same is shown in (b), but for $C=0$. In this case, the average conductance always obeys a power law. The inset shows that the exponent $\beta$ increases monotonically with $\alpha$.

shows that the exponent $\beta$ vanishes for small values of $\alpha$, meaning that the average conductance becomes independent of $N$. However, as $\alpha$ increases, the conductance exponent approaches unity, the analytical result for a regular one-dimensional lattice.

Going back to the optimal flow condition, namely, $C=1$ and $\alpha=1$, since logarithmic corrections are also present, an exponent $\beta \rightarrow 0+$ is expected. To support this conjecture, we show in Fig. 4 that the average conductance in this particular case follows a power law of the logarithm of $N,\langle G\rangle \propto\left(\log _{10} N\right)^{-\gamma}$, with an exponent $\gamma=2.16 \pm 0.02$.

Next, we investigate the optimal flow conditions for small-world networks built over two-dimensional substrates, namely, $L \times L$ square lattices. In the case where all local conductances are equal, $C=0$, we obtain $\alpha_{\text {opt }}=0$ (see Fig. 1 of the Supplemental Material [33]), which is compatible with the behavior previously observed for one-dimensional substrates (see the inset of Fig. 2).

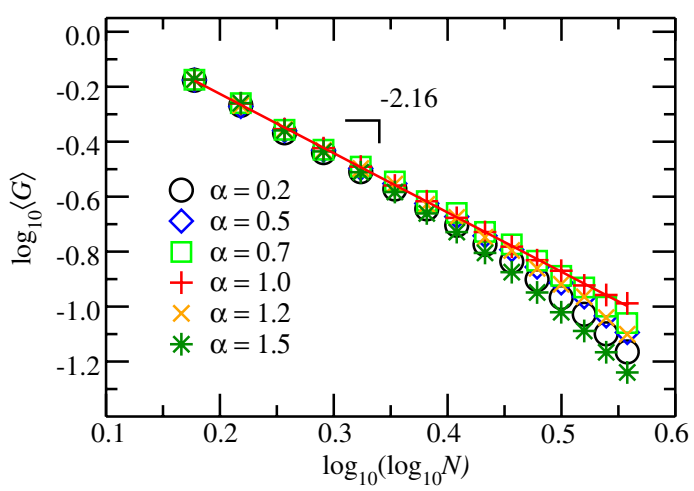

FIG. 4 (color online). For $C=1$ and $\alpha=1$, the global conductance follows a power-law of the logarithm of $N$, $\langle G\rangle \propto\left(\log _{10} N\right)^{-\gamma}$. The least-squares fitting to the data points for $\alpha=1$ gives an exponent $\gamma=2.16 \pm 0.02$.

As displayed in Fig. 5(a), our results show that, in the case where the local conductance is inversely proportional to the link length, $C=1$, the maximum values of the average network conductance $\langle G\rangle$ are obtained at $\alpha \approx 2.7$
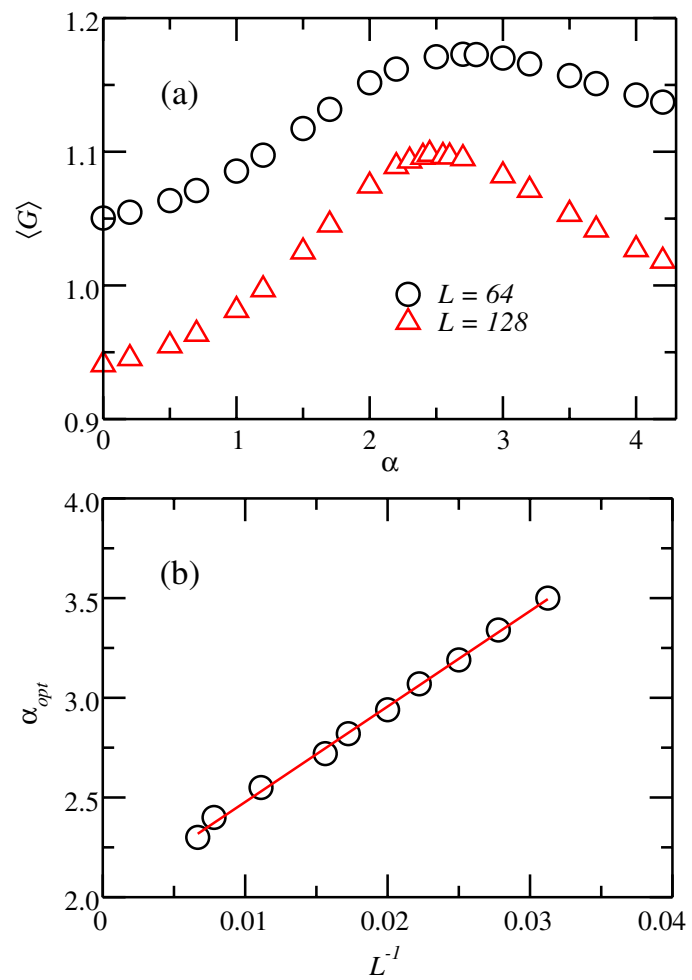

FIG. 5 (color online). In (a), we show the dependence on $\alpha$ of the average global conductance $\langle G\rangle$ of networks built by adding long-range connections on two-dimensional regular lattices, for the case of $C=1$. The maximum values of $\langle G\rangle$ are obtained at $\alpha \approx 2.7$ for $L=64$, and at $\alpha \approx 2.4$ for $L=128$. For all points, the error bars are smaller than the symbols. The dependence of $\alpha_{\mathrm{opt}}$ on $1 / L$ is shown in (b). The linear regression (red solid line) to the data points suggests that $\alpha_{\text {opt }} \rightarrow 2$ as $L$ increases according to $\alpha_{\text {opt }}=1.998+47.873 L^{-1}$. For each value of $L, \alpha_{\text {opt }}$ is obtained with a relative error equal to $10^{-2}$. 
for $L=64$, and at $\alpha \approx 2.4$ for $L=128$. In Fig. 2 of the Supplemental Material [33], we present maps with the spatial distributions of effective conductances between the central node and every other node in typical networks of size $L=128$, calculated for different values of $\alpha$. The plot for $\alpha=2.4$ notably shows increased values of conductance over the whole network. However, the finite-size scaling analysis shown in Fig. 5(b) clearly indicates that $\alpha_{\text {opt }} \rightarrow 2$ as the system size $L$ increases. More precisely, the linear regression of $\alpha_{\mathrm{opt}}$ versus $1 / L$ gives $\alpha_{\mathrm{opt}}=1.998+$ $47.873 L^{-1}$. These results for two-dimensional substrates, together with those for one-dimensional ones, give support to the conjectures that $\alpha_{\mathrm{opt}}=0$ for $C=0$, and $\alpha_{\mathrm{opt}}=d$ for $C=1$.

To explore more deeply the phenomenon beyond the Pouillet's law $(C=1)$, we now study in detail the dependence on the parameter $C$ of the average global conductance calculated for networks built on one-dimensional substrates. Note that, in the absence of long-range connections, the effective conductance between any pair of sites grows linearly with the distance $r$. That is, for $C>1$, these effective conductances are always larger than the conductances of their long-range connections in parallel. In this case, therefore, long-range connections do not play the role of shortcuts. For $C>0$, the longer the added connections, stronger should be their effect on the global conductance. On the other hand, the longer the connections, more resistive they are, with less impact on the effective conductance. These competing effects are responsible for the observed nonmonotonicity of the average conductance with the exponent $\alpha$. Such a conclusion is supported by the results shown in Fig. 6, where an optimal $\alpha$ is observed for $0.2 \leq C<1.8$. We cannot, however, exclude the possibility that finite size effects are hiding the optimum condition in $C=1.8$.

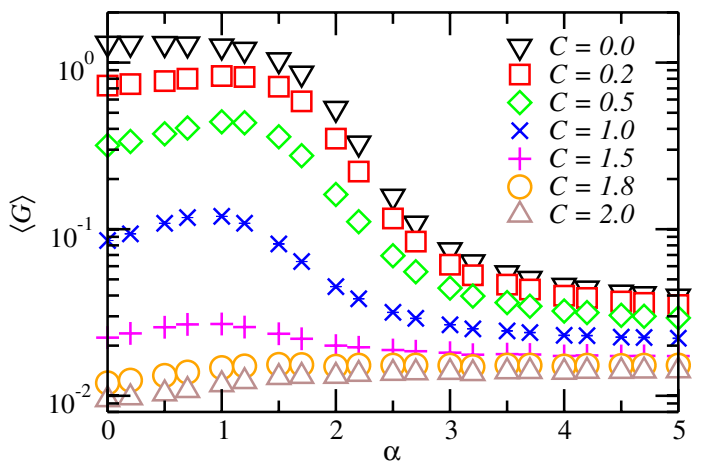

FIG. 6 (color online). Dependence on $\alpha$ of the average global conductance of the network for different values of $C$. The underlying substrate is a one-dimensional regular lattice with $N=2048$. The optimal global conductance is found for three different regimes of $C$ : for $C=0, \alpha_{\text {opt }}=0$; for $0.2 \leq C<1.8$, $\alpha_{\text {opt }}=1$; and for $C \geq 1.8$, the conductances always grow with $\alpha$; namely, the optimal condition is $\alpha \rightarrow \infty$.
Our results present an striking connection with the problem of navigation in small-world networks. There we have $\alpha_{\mathrm{opt}}=0$ for global knowledge [24] and $\alpha_{\mathrm{opt}}=d$ for local knowledge [25]. Here we obtain the same optimal conditions for equal link conductances and for conductances that decrease with the link length, respectively. In a sense, solving Kirchhoff's laws involves more global knowledge than finding the minimum path, since the flux balances are susceptible to small disturbances, like the addition or removal of a single conducting link anywhere in the lattice. Therefore, it is somewhat surprising that we obtain for $C=1$ the same optimal condition as in the case of navigation with local knowledge. The key feature of this result is the way the parameter $\alpha$ controls the length of long-range connections. If $\alpha$ is small, long connections become frequent. However, since $C>0$, their associated conductances are low. On the other hand, if $\alpha$ is too large, the conductances of added connections do not vanish, but their lengths are too small to impact the scaling.

In conclusion, our results showed in what conditions enhanced flow can be observed in small-world networks. Long-range interactions are known to strongly affect the physical properties of real systems. Often the amount of these interactions is parametrized either by considering a vanishing strength for the interaction or a vanishing probability for establishing it. Here we considered the combined effect of these two conditions by associating power laws for both $(i)$ the probability distribution of distances for long-range links, $P_{i j} \sim r_{i j}^{-\alpha}$, and (ii) their corresponding conductances, $g_{i j} \sim r_{i j}^{-C}$. For $C=0$, the longer the link, the stronger its impact on the flow, leading to $\alpha_{\text {opt }}=0$. For $C>2$, longer random links have decreasingly small conductances. In this regime, increasing the probability of longer connections is detrimental to the conductivity, and we obtained $\alpha_{\mathrm{opt}} \rightarrow \infty$. For intermediate values, $0<C \ll 2$, we observed an optimal condition at $\alpha_{\mathrm{opt}}=d$. Interestingly, the same optimal conditions were verified for the problems of navigation with global knowledge [24], $\alpha_{\mathrm{opt}}=0$, and local knowledge [25], $\alpha_{\mathrm{opt}}=d$. Moreover, in the case of Pouillet's law, $C=1$, we observed that, in the optimal condition, the conductance vanishes slowly with the size of the system $\langle G\rangle \propto\left(\log _{10} N\right)^{-\gamma}$, with $\gamma=2.16$ for one-dimensional substrates. Finally, it is interesting to note that the conductance between two sites $\mathrm{A}$ and $\mathrm{B}$ in a network, for $C=0$, corresponds to the escape probability of a random walk, i.e., the probability that a diffusing particle, starting at $A$, reaches $B$ before returning to $A$ [34]. Therefore, our results for $C=0$ can also be used to interpret first-passage processes of random walks through networks.

We thank the Brazilian agencies CNPq, CAPES, FUNCAP, and FINEP, the FUNCAP/CNPq Pronex grant, and the National Institute of Science and Technology for Complex Systems in Brazil for financial support. 
*lucas@fisica.ufc.br †pablo@fisica.ufc.br \#auto@fisica.ufc.br

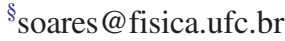

[1] B. Bollobás, Modern Graph Theory (Graduate Texts in Mathematics) (Springer, New York, 2012).

[2] R. Monasson, Eur. Phys. J. B 12, 555 (1999).

[3] J. S. Andrade, D. A. Street, Y. Shibusa, S. Havlin, and H. E. Stanley, Phys. Rev. E 55772 (1997).

[4] C. Conti and S. Trillo, Phys. Rev. Lett. 92, 120404 (2004).

[5] G. Beylkin and M. J. Mohlenkamp, Proc. Natl. Acad. Sci. U.S.A. 99, 10246 (2002).

[6] B. Kahng, G. G. Batrouni, S. Redner, L. de Arcangelis, and H. J. Herrmann, Phys. Rev. B 37, 7625 (1988).

[7] A. A. Moreira, C. L. N. Oliveira, A. Hansen, N. A. M. Araújo, H. J. Herrmann, and J. S. Andrade, Phys. Rev. Lett. 109, 255701 (2012).

[8] C. L. N. Oliveira, A. P. Vieira, H. J. Herrmann, and J. S. Andrade, Europhys. Lett. 100, 36006 (2012).

[9] Y. Lee, J. S. Andrade, S. V. Buldyrev, N. V. Dokholyan, S. Havlin, P. R. King, G. Paul, and H. E. Stanley, Phys. Rev. E 603425 (1999).

[10] S. Kirkpatrick, Phys. Rev. Lett. 27, 1722 (1971); D. J. Thouless and S. Kirkpatrick, J. Phys. C 14, 235 (1981).

[11] V. V. Cheianov, V. I. Fal'ko, B. L. Altshuler, and I. L. Aleiner, Phys. Rev. Lett. 99, 176801 (2007).

[12] M. E. J. Newman, M. Girvan, Phys. Rev. E 69, 026113 (2004).

[13] G. Korniss, M. B. Hastings, K. E. Bassler, M. J. Berryman, B. Kozma, and D. Abbott, Phys. Lett. A 350, 324 (2006).

[14] E. López, S. V. Buldyrev, S. Havlin, and H. E. Stanley, Phys. Rev. Lett. 94, 248701 (2005).

[15] S. Çalişkan, M. A. Novotny, and J. I. Cerdá, J. Appl. Phys. 102, 013707 (2007).

[16] D.-S. Lee and H. Rieger, Europhys. Lett. 73, 471 (2006).

[17] D. Helbing, D. Armbruster, A. S. Mikhailov, and E. Lefeber, Physica (Amsterdam) 363A, xi (2006).
[18] B. Danila, Y. Yu, J. A. Marsh, and K. E. Bassler, Chaos 17, 026102 (2007).

[19] J. S. Andrade, H. J. Herrmann, A. A. Moreira, and C. L. N. Oliveira, Phys. Rev. E 83, 031133 (2011).

[20] R. Albert and A.-L. Barabási, Nature (London) 401, 130 (1999).

[21] D. Watts and S. Strogatz, Nature (London) 393, 440 (1998).

[22] M. E. J. Newman and D. J. Watts, Phys. Rev. E 60, 7332 (1999).

[23] C. F. Moukarzel, M. Argollo de Menezes, Phys. Rev. E 65, 056709 (2002).

[24] K. Kosmidis, S. Havlin, and A. Bunde, Europhys. Lett. 82, 48005 (2008).

[25] J. M. Kleinberg, Nature (London) 406, 845 (2000).

[26] G. Li, S. D. S. Reis, A. A. Moreira, S. Havlin, H. E. Stanley, and J. S. Andrade, Phys. Rev. Lett. 104, 018701 (2010); Phys. Rev. E 87, 042810 (2013).

[27] M. Barthélemy, Phys. Rep. 499, 1 (2011).

[28] L. K. Gallos, H. A. Makse, and M. Sigman, Proc. Natl. Acad. Sci. U.S.A. 109, 2825 (2012).

[29] Although we use the term long range generically, the length of the added connection may be small, varying from 2 to $\mathrm{N} / 2$.

[30] The resulting systems of linear algebraic equations were solved through the HSL library, a collection of FORTRAN codes for large-scale scientific computation. See http://www .hsl.rl.ac.uk/.

[31] Due to the large amount of stored data, which can easily reach hundreds of gigabytes, we average the conductance over $10 \%$ of all possible pairs of sites in the network, randomly chosen in each realization.

[32] N. Kipnis, Sci. Ed. 18, 349 (2009).

[33] See Supplemental Material at http://link.aps.org/ supplemental/10.1103/PhysRevLett.112.148701 for additional results on flow in small-world networks built over two-dimensional substrates.

[34] P. G. Doyle and J. L. Snell, Random Walks and Electric Networks, Carus Monographs Series (Mathematical Association of America, Oberlin, OH, 1984). 